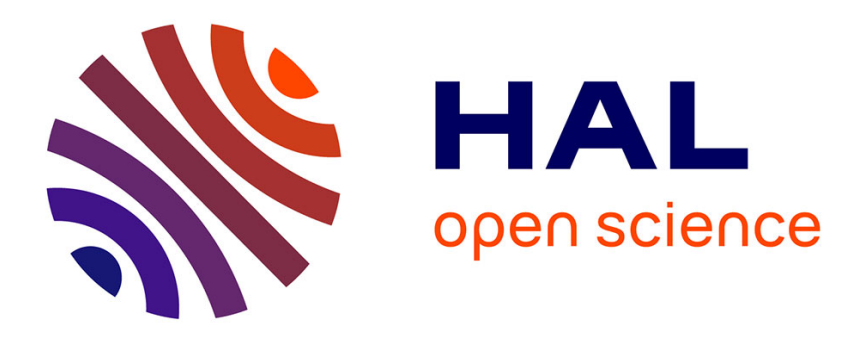

\title{
Literature Review Toward Decentralized Railway Traffic Management
}

\author{
Elisa Marcelli, Paola Pellegrini
}

\section{To cite this version:}

Elisa Marcelli, Paola Pellegrini. Literature Review Toward Decentralized Railway Traffic Management. IEEE Intelligent Transportation Systems Magazine, 2020, 18p. 10.1109/MITS.2020.2970180 . hal02617135

\section{HAL Id: hal-02617135 \\ https://hal.science/hal-02617135}

Submitted on 25 May 2020

HAL is a multi-disciplinary open access archive for the deposit and dissemination of scientific research documents, whether they are published or not. The documents may come from teaching and research institutions in France or abroad, or from public or private research centers.
L'archive ouverte pluridisciplinaire HAL, est destinée au dépôt et à la diffusion de documents scientifiques de niveau recherche, publiés ou non, émanant des établissements d'enseignement et de recherche français ou étrangers, des laboratoires publics ou privés. 


\title{
Literature Review Toward Decentralized Railway Traffic Management
}

\author{
Elisa Marcelli and Paola Pellegrini
}

IEEE Intelligent Transportation Systems Magazine

2020 


\title{
Literature Review Toward Decentralized Railway Traffic Management
}

\author{
Elisa Marcelli and Paola Pellegrini
}

\begin{abstract}
This paper analyses the literature to identify ideas which may be applied to decentralized real-time railway traffic management. This system represents a new way for dealing with railway traffic perturbations in absence of a central decision maker. Specifically, we are interested in identifying techniques that may constitute suitable automatic mechanisms for the emergence of an effective system behaviour. In this literature review, we discuss the possibility of exploiting the existing research works on other transport modes. The analysis of these works makes it clear that real-time railway traffic management is very peculiar. Hence, we consider different approaches: hierarchical self organization, task allocation, reinforcement learning, consensus, auction and coopetition techniques. Some promising possibilities emerge, which we analyse proposing ideas for modelling decentralized real-time railway traffic management.
\end{abstract}

Index Terms-Real-time railway traffic management, Multiagent system, Hierarchical self-organization, Task allocation, Reinforcement learning, Consensus, Auction, Coopetition

\section{INTRODUCTION}

$\mathbf{T}$ RAFFIC management describes the process of coordinating, controlling and organizing traffic to achieve efficiency and effectiveness in the use of existing infrastructure capacity. In the overall traffic management problem, some months before operations, a timetable is produced. This timetable defines speeds, precedences and routes so that trains can travel never encountering congested traffic conditions. However, when an unexpected event perturbs operations causing the delay of one or more trains, trains travelling at the planned speed may concurrently require the use of some parts of infrastructure, which corresponds to the emergence of conflicts. When this happens, the planned timetable becomes infeasible and an effective alternative must be determined. Finding this alternative is the objective of real-time railway traffic management problem [66]: it seeks for the redefinition of precedences and routes to minimize delay propagation. In the literature, it is also referred to as train rescheduling or traffic control. In this paper we use the wording realtime railway traffic management to stress that train rerouting is part of the problem we consider, and avoid confusion with term train control, which is typically used for problems related to speed profiles definition [41]. Currently, real-time railway traffic management is performed by dispatchers in a centralized way: a dispatcher is in charge of all traffic traversing a control area.

E. Marcelli is with the Department of Mathematics, School of Sciences and Technology, University of Camerino, via Madonna delle Carceri 9, Camerino (MC) Italy (e-mail: elisa.marcelli@unicam.it).

P. Pellegrini is with Univ. Lille Nord de France, F-59000 Lille, IFSTTAR, COSYS, LEOST, F-59650 Villeneuve d'Ascq, France (e-mail: paola.pellegrini@ifsttar.fr).
Following current practice, all decision support tools proposing optimization techniques for traffic management consider a centralized decision making. Here, decisions are made and imposed to trains so that a system-level utility function is optimized. This utility function is typically related to delay propagation and is then to be minimized.

In this paper, we consider a radically different approach for real-time railway traffic management, in which decisions are not made in a centralized way: either the system is driven by an emergent beaviour, or trains themselves make decisions on how to solve conflicts, choosing precedences and routes. These trains are intelligent and autonomous for what concerns vehicle to vehicle and vehicle to infrastructure communication, on the one hand, and priority and routing decision making, on the other hand. Indeed, they may be autonomous also as for driving, in this case being automated. However, we consider the driving aspects independent from real-time traffic management, as well as communication ones. We only focus on intelligent trains capable of real-time traffic management decision making: a driver may or may not be present onboard, and communication is supposed to be completely reliable. Undoubtedly, even in a decentralized system as the one we consider, railways always require the presence of a central traffic management. This will be in charge of tackling situations that cannot be solved by intelligent trains alone. For example, in case of major disruptions as the unavailability of a part of the railway network for some hours requiring shortturnings and cancellations, the decision making must follow specific steps and needs to be centralized [10]. However, today's system functioning and technological capabilities allow thinking of intelligent trains taking the burden of real-time management of minor perturbations that generate conflicts and occur regularly in the network.

In countries in which infrastructure use and train running are managed by the same organization, passing from centralized to decentralized real-time railway traffic management may be seen as a simple movement of decision making at different levels of the organization hierarchy. Centralized decision making is concentrated at a single level of this hierarchy [13]. In some sense, one may visualize the railway system as hierarchical even when organizations are different. Here the infrastructure manager may be at the top of the pyramid being in charge of the overall network, railway undertakings in the middle and individual trains at the bottom. However, this visualization may definitely be arguable, and we will not consider it in the rest of this paper. In any case, in non-centralized processes, decision making can also be dispersed across different levels of an organization, or across different actors or stakeholders if 
several organizations are involved. In some sense, the situation of having major disruptions managed centrally while minor perturbations are tackled by trains may be interpreted as an example of dispersed decision making. However, we only consider here the case of minor perturbations, for which we claim that the leadership of the real-time traffic management process can be effectively and completely shared among trains. In [13] this is referred as decentralization, which is then the wording we will use throughout this work. Hereinafter, we will only focus on the literature related to decentralization as defined here, neglecting other approaches which may concern dispersion or fragmentation of decision making. Although connected, these approaches are out of the scope of this paper, in which we consider a well defined problem that results from various discussions with railway actors. To the best of our knowledge, anyway, no major hints from other decision making strategies are available for real-time railway traffic management.

In the following, we propose a literature review of possible ways for defining such decentralized system in scheduling or allocation problems. From this review, we try to identify possible ways of modelling such a system for real-time railway traffic management.

The rest of the paper is organized as follows. Initially, in Section II, we summarize the state of the art on centralized real-time railway traffic management, and we indicate the main lacks of the existing approaches which may be overcome in a decentralized system. Then, in Section III, we report the very little existing literature on decentralized real-time railway traffic management. In Section IV we analyse research works on decentralized real-time traffic management in other modes of transport. Namely, we consider road traffic, shared mobility, air traffic and waterways traffic management. This analysis clarifies that the problems tackled in the literature for other modes are different and not really compatible with the one we are interested in for railway. Specifically, major differences exist in the need for collaboration and agreement in general, as well as in the system constraints. In the rest of the paper, we focus on the more general literature concerning these needs. In Section V, we describe some different approaches that may be used for modelling a decentralized system of intelligent trains, and we discuss which possibilities seem the most appropriate. Finally, in Section VI we draw conclusions.

\section{STATE OF THE ART ON CENTRALIZED REAL-TIME} TRAFFIC MANAGEMENT

The real-time railway traffic management problem, in its centralized version, is the object of a large body of literature. Quite comprehensive reviews have been published [11], [16].

A variety of combinatorial optimization approaches have been applied to this problem, from integer linear programming [55], [66], [93] to alternative graphs [21], [54], to heuristics and meta-heuristics [15], [78]. Also a reinforcement learning approach has been proposed [82]. Here, the centralized decisions are made by a simulator, which learns to discriminate between good and bad scheduling solutions in terms of delay propagation. The approach is implemented in a trivial example considering three trains and three stations and the results show that reinforcement learning may be used as an efficient alternative to classic optimization methods. All these approaches make decisions on all trains present in a control area over a predefined time horizon. A remarkable exception is represented by [89]. The paper proposes a way for selecting a smaller set of trains by identifying what the authors call dynamic impact zone for every conflict to be tackled. Then, a centralized decision is made concerning all these trains.

These approaches are aimed at being part of decision support tools for dispatchers, as tested in the ON-TIME European project with the collaboration of railway infrastructure managers [63], [70]. However, the actual deployment of such decision support tools is at its very early stages. Few successful implementations can be identified, as the Trento-Bassano del Grappa line in Italy, the Stavanger-Moi line in Norway, or a network of five lines in Latvia. Unfortunately, the performance achieved and the lessons learned from the development and implementation of these systems are only partially available to the community [49].

Undoubtedly, tackling the problem centrally allows the consideration of the impact of decisions on the whole control area before selecting the final ones. Hence, no myopic decisions are made: decisions that look good when they are made but having problematic effects in the following. In this sense, the performance achieved by a centralized approach can hardly be achieved by a decentralized one: the latter is myopic by definition, decisions being based on local information. Indeed, when management is distributed across many independent, loosely coupled subsystems, as the decentralization approach defined in [1], the intelligent vehicle makes decisions using local measurements and optimizes local dynamics.

However, three main challenges still need to be faced. They are particularly critical in centralized approaches and may be at least partially overcome in decentralized ones. First, there are limits to the size of the control areas that may be considered in centralized approaches. Despite the effectiveness of the algorithms and the better and better performance of computers, it will always be possible to define a case study too large to be tackled. Indeed, in today's context, in which a single European railway area is aimed at [67], the actual problem of real-time railway traffic management is larger than what can be thought to ever be solvable in one shot. Few papers try to face the challenge of size limits, either decomposing a large network in control areas to be coordinated [17], [19], or proposing algorithms capable of treating larger networks than what was done before [48]. However, this challenge is still open and there is no clearly identified research direction for overcoming it. Indeed, when moving to a decentralized system, in which decisions are made based on more or less extended local knowledge, this challenge would be implicitly solved. Moreover, if large case studies were to be tackled, the gap in performance between centralized and decentralized approaches would become thinner and thinner. In fact, a centralized approach might struggle due to the size of the case study and the short computational time available due to the real-time nature of the problem. Thus, despite its myopic decisions, the decentralized approach might be able to close 
the gap.

The second challenge is linked to the robustness of the system to computer or communication failures. Indeed, in case of a failure, at least a part of the system will always be blocked. However, with a centralized approach, the whole control area where the failure occurs will be touched. Instead, failures would impact only the immediate vicinity of the interested area with a decentralized approach.

Third, the railway market is today open to competition [4]. Indeed, with the exception of the UK, today national markets in Europe are mostly covered by the incumbent railway undertaking, which has very strong links with the infrastructure manager in charge of real-time railway traffic management. In a centralized system, then, there might be entrance barriers due to the need of disclosing private information considered competition-critical with the infrastructure manager, which is an external entity and may be perceived as non completely neutral. If a suitable system was designed, decentralizing decisions would somehow favour competition between railway undertakings, avoiding the need of revealing private information.

\section{STATE OF THE ART ON DECENTRALIZED REAL-TIME TRAFFIC MANAGEMENT}

In most of the existing literature, when real-time railway traffic management is said to be tackled in a decentralized way, the system is actually split into subsystems, each one relying on one individual or tool to make decisions and provide directions [17] [19] [44]. Therefore, traffic in a railway infrastructure is in general not really managed in a decentralized way but rather a disperse one: it is simply partitioned into geographically smaller areas where a centralized management is in place.

To the best of our knowledge, very few papers deal with an actual decentralized system.

In [98], decentralized real-time railway management is achieved through swarm intelligence: trains that are locally moving in the same direction and facing a possible common conflict are grouped into a swarm. To coordinate with its neighbours in the swarm, each train uses a train-to-train communication system. To occupy a track, trains have to send the request to a decentralized dispatching algorithm which verifies the feasibility of such request. If the requested track is free, the algorithm allows the train to occupy it, otherwise the request is put in a pending list. Although this paper presents an innovative idea, it represents a theoretical starting point that does not give tangible solutions. For example, no investigation is made on the mechanism to define and adapt swarm compositions in complex networks and throughout time. Moreover the dispatching algorithm, which in our eyes constitutes the most important part of the approach, is only sketched.

An approach using autonomous agents is proposed in [33]. The paper describes how agents could be used for both planning long term schedules and making short term changes when needed. A peculiarity of this work is that agents represent trains and tracks: when a journey has to be planned, the considered train (T-agent) creates a list of all possible routes it may take, ordered in terms of its own priority criteria, and starts negotiating with tracks (S-agents) the cost of using them. If the cost is too high for all the possible routes, the journey is cancelled. In this system, the interest of the T-agent is finding the more suitable route at a convenient price; at the same time, S-agents aim to fix costs so as to satisfy as many trains as possible to maximize revenues. Hence, both T-agents and S-agents have an active role in the negotiations. As [98], this paper proposes an innovative idea but does not deepen it, remaining a hypothetical notion. Specifically, no explanation is attempted to define track costs, for example in case of competition. Moreover, only one to one negotiation is envisaged, neglecting traffic conditions even in real time. In any case, no proper algorithm for the negotiation is discussed.

A decentralized model is also presented in [83]. The paper analyses the problem of dealing with train organization presuming that trains are capable of self-organizing skills and communicating with one another. Specifically, a finite set of trains moving on a loop railway line is considered, where each train has the capability of communicating (i.e., receiving information) only with one train ahead. The behaviour of each train is decided solving a local optimization problem aiming to minimize its time deviation with respect to the scheduled time, having as result two sequences: one defining its future control strategy and the other establishing its future departure timeline. Even if this work proposes a very interesting approach, it is defined by an essential feature: each train has to solve a minimization problem only with the information in its possession and the one coming from its predecessor. Independently of the infrastructure and propagation of information, in such a framework any decision of a train will always myopic. Indeed, the paper is restricted to small and linear systems in which such myopic decisions guarantee the attainment of feasible and possibly good traffic management solutions. Unfortunately, this is not the case of a common railway system.

Decentralized hierarchical approaches are proposed in [69] and [39]. They exploit the concept of multi-agent system (MAS). A MAS [34] is an aggregation of agents, intelligent autonomous entities with the capability of observing the environment, communicating with each-other and finally making decisions based on these observations and communications. [69] presents a MAS in which real-time railway traffic management is performed at three communicating levels. A supervisor level makes decisions on trains' precedence; a train level makes choices on driving profiles, given the supervisor's decisions; a station level gives orders for trains arrivals and departures and provides information to users. A learning process is also introduced, thanks to which the supervisor uses past situations to infer rules that anticipate conflicts. In this work, the railway infrastructure representation is quite abstract, omitting any consideration of the actual details of its functioning. Another three-level approach based on MAS is described by [39]. Here, train agents are at the bottom, station agents are in the middle, and a traffic control center agent is at the top level. The amount of information on the whole system increases across these levels, passing from limited for trains, to local for stations, to global for the traffic control center. 
Higher levels intervene if lower levels do not manage to find a feasible solution when perturbations occur. The paper says that a negotiation may be put in place to establish precedences between trains, but no approach is proposed to do so.

Very recently, [45] applies reinforcement learning to the decentralized real-time railway traffic management problem. Here, the system automatically learns what choices bring to good performances, and tries to replicate them whenever suitable. This work defines an original approach for a linear network in which bidirectional lines are represented mesoscopically: some details of the actual infrastructure are neglected. For example, only one train at a time can be on the track between two stations. Train schedules are defined in terms of departure times from stations, hence considering the so-called green-wave strategy [18]: trains cannot stop along the line to give precedence to one another, and junctions are crossed in a first-come-first-served way. In the proposed reinforcement learning scheme, a delay threshold is defined. If with the selected schedule the sum of delays is less than such threshold, the system gets a reward, otherwise it suffers a penalty.

Only the last work constitutes a basis for the decentralized system we have in mind, which may be able to replace the centralized decision making process in place today for the railway network. Indeed, several aspects must necessarily be considered. In particular, once trains detect a conflict emerging ahead, they have to decide whether to locally reroute, e.g., changing the platforms used to stop at a station with respect to the original plan, or to modify their passing order with respect to other trains. Several trains may be involved in the conflict, belonging to one or several railway undertakings. If they belong to the same railway undertaking, reaching an agreement may be easy, because defining the priority of services may be immediate. Instead, if several railway undertakings are involved, they may be willing to sacrifice some trains to favour others, without necessarily wanting to share the reasons of their choice. Moreover, the utility that a train may associate to the passing order at a junction is in principle linked to the possible outcomes of other conflicts at junctions it will cross later on. Indeed, trains it may enter in conflict with in future junctions may be different from the current ones. The decisions then should not be completely independent.

\section{DECENTRALIZED TRAFFIC MANAGEMENT IN OTHER MODES}

As reported in Section III, decentralized approaches for realtime railway traffic management are very rarely found in the literature.

Differently, more works exist for other means of transport. In this section, we discuss four of these modes, highlighting similarities and differences with respect to the problem we consider in this paper. Specifically, we analyse road traffic, shared mobility, air traffic and waterways traffic management.

\section{A. Road Traffic Management}

Approaches for decentralized traffic management have been widely studied in the field of road transport. The number of vehicles moving on road or public highway has significantly grown in the last years and traffic congestion has become a major issue in many large cities and crowded areas. To analyse this issue, many works regarding the idea of constructing simulation models for road traffic management have been presented [9] [29] [51]. These models describe the use of MAS to deal with vehicles and infrastructures, with the goal of testing principles and making useful traffic control plans of action.

In [29] it is described the use of reactive agents in controlling road vehicles in a simulated traffic environment. Each driving agent receives external information about the environment through sensors and, based on these, makes its own decisions. The behaviour that an agent applies is given by a set of rules which describes actions it shall carry out in order to keep a civil conduct, e.g., always driving on the streets or decelerating when a crossroad is met. These rules are influenced by several behaviour parameters with the aim of creating different driving styles, as happens in real life. In this way, it is possible to let some vehicles drive more or less aggressively, or regulate their speed as preferred, making every agent in some way dissimilar from each other. Indeed, this system may be seen as a self-organized road traffic management one.

In [25], agents are used to study systems to primarily reduce traffic jams in crossroads. As before, the system is decentralized and each agent can autonomously make its own decisions submitting to road rules. The paper proposes a reservation-based procedure to be used instead of traffic lights, so that vehicles have time gaps during which they are allowed to occupy the crossroad. Reservations are made on a firstcome first-served basis, i.e., the first vehicle which demands the permission to cross the intersection at a certain time has its reservation accepted. The advantage of this method with respect to a simple first-come first-served approach consists in the possibility for vehicles to adapt their speed to the reservation they obtain. As reported in [75], a noticeable amount of studies focus on mechanisms for autonomous vehicles to cross intersections. Many of them are based on decentralized decision making. However, differently from the railway traffic management problem we consider, the mechanisms have the objective of minimizing the intersection crossing time while ensuring safety. To the best of our knowledge, no consideration of vehicle utilities is ever mentioned.

A policy for collision control among multiple vehicles is given in [64]. The article uses MAS to study the cooperation between several vehicles on the same route, with the possibility of leaving or entering the scene at any time, focusing on collision avoidance. More recently, [77] solves the same problem with a combination of optimal control and sequential decision making. The paper shows how to use tools from reachability theory for obtaining model-based heuristics capable of coordinating vehicles.

To tackle congestions in the network rather than in a crossroad, [14] proposes a MAS approach. Specifically, agents deal with congestions before actually meeting other vehicles, by making use of traffic density forecast. Agents are divided into driving and infrastructure agents, and they move in a virtual environment representing physical roads and junctions. 
The focal point is that driving and infrastructure agents do not communicate with each other. To make the bridge among them, the paper uses delegate or lightweight agents, named ant-agents. They can follow two possible behaviours: either they explore their neighbouring areas, looking for traffic congestion, or they are intention ants, providing infrastructure agents information on the routes driving agents aim to use. Note that the name ant-agent comes from the fact that these agents behave similarly to ants looking for food and leaving pheromones behind when they reach a food source. In this case, pheromones represent traffic information to be used by other ant-agents: the longer an agent takes to traverse a route, the more pheromones it deposits, giving local information about traffic jam.

The same objective but with a completely different perspective is pursued by [74]. Here, traffic management does not emerge from the behaviour of vehicle-agents that need to coordinate. Instead, each external traffic controller operates on a part of the network and need to make coherent traffic management decisions. The main objective is minimizing the total amount of time that vehicles spend travelling through the network in a predefined time horizon. The proposed optimization approach dynamically recognizes which traffic controllers should be put in the same cluster (i.e., controller influencing and having impact on each other) and optimized together, and when it is not appropriate to do so.

MAS are also used to study cooperative control in road traffic management, namely the coordination of a group of vehicles that travel on the same route. For example, the collaboration between autonomous vehicles is the central topic of [32]. The main goal of the paper is to get a control approach of the formation of groups of agents, robust to communication network changes.

The works reported in this section show how agents are used in road traffic to model cars' selfish acting: cars are not directly controllable units of the system. On the opposite, at least up to a certain degree, trains are directly controllable by dispatchers, and to some extent must remain such. Hence, they cannot necessarily act in a selfish way, but must pursue somehow the good performance of the overall system. Therefore, the analysed approaches cannot be exported to model the realtime railway traffic management system: the export would only make sense if cars were modelled as agents aiming for the overall optimal traffic flow. Indeed, the transfer of ideas from road traffic to railway traffic is far-fetched, due to the fundamental differences between the two systems.

Moreover, even if there were approaches aiming at this overall optimality, a further difference between road and railway traffic must be taken into account. Specifically, in road traffic an attitude called human error must be considered [23], where failures must be analysed to understand and predict car driving violations. In this regard, some works focus on the importance of driver violations in the case of road traffic simulation. For example, in [24] agents are used to describe a simulation, taking as a model real drivers' behaviour. The aim of the article is to make the simulation as realistic as possible, focusing on some features that are an important part of real driver decision making processes. In particular, the model relies on opportunistic strategies. In railway traffic, opportunistic strategies cannot be accepted. For example, in road traffic, violations of safety distance between cars often occur. In railway, these violations are not allowed: the system automatically triggers emergency braking if a train manages to get too close to the preceding one. In this sense, no flexibility is possible, and any decentralized real-time traffic management approach does not need to take care of it.

\section{B. Shared Mobility Management}

The term shared mobility identifies transfers from one place to another which take place with shared vehicles: users do not exploit their own cars, bicycles or bikes, instead they use rental services paying for the vehicle according to a predefined rental rate. Shared mobility has grown tremendously in the past decade both because of its increasingly competitive prices and efficiency but also for its environmentalist facet: it decreases traffic congestion and reduces several forms of pollution. From a business point of view, shared mobility could spread also thanks to the birth of many companies and platforms offering transfer services. Anyway, a variety of concerns arise linked to this newborn system.

A solid approach for shared mobility using a reinforcement learning algorithm is proposed in [38]: vehicles are modelled as autonomous agents, giving advices when a driver is in control and directing otherwise, learning how to behave by using rewards. The paper seeks to tackle two main problems: assigning vehicles to users and performing vehicle rebalance, i.e., redistributing idle vehicles to high demand areas. An autonomous vehicle is rewarded each time it has a positive behaviour, specifically whenever it serves pending requests or it moves to a highly requested empty area. The main objective is to maximize such rewards and this drives the learning mechanism: the vehicle observes the reward obtained as a consequence of its behaviour and tends to replicate what previously resulted to be benefiting.

An interesting method for decentralized shared mobility is presented in [79] and is based on the idea of co-utility. The term co-utility defines a strategy of cooperation between more than one participant, where each participant utility maximization is linked also to the increase of other participants utility. One of the key principles of co-utility is that "helping others is the best rational option even if players are selfish". The paper proposes a decentralized system which may reduce two of the main concerns when dealing with shared mobility. First, it may reduce the lack of trust, i.e., the fact that users may not appreciate sharing a vehicle with strangers. Second, the privacy concern linked to the sharing of personal information. In such system, drivers and passengers may interact without any central authority checking on exchanges. The system may be controlled by some kind of reputation technique ensuring fairness. Each user is assigned an identification number and, for a vehicle sharing to take place, driver and passenger users have to publish advertisements compatible with each other (i.e., route, time, geographical zone).

All the works on decentralized shared mobility management, exemplified by the ones presented in this section, aim to 
the coordination of vehicles and users each having a personal objective. This perspective cannot be transferred to real-time railway traffic management, where trains must try to stick as much as possible to an existing timetable. However, the idea at the basis of co-utility in which the utility of a system component depends on the one of other components as well, and hence somehow on the performance of the system, may be interesting, as will be mentioned in Section V-F.

\section{Air Traffic System}

Air Traffic Management (ATM) deals with all functions necessary for safety and efficiency of movements during all phases of a flight, starting from the departure from an airport, considering all intermediate flight phases, until arrival at destination airport. Nowadays, the coordination of aircraft movements is done through centralized ground-based systems: decisions are made centrally by air traffic controllers and imposed to aircraft. However, several research works deal with the idea of a less and less centralized control system. They are motivated by the growing development of the automation field and by the ever-increasing number of aircraft flying every day, which significantly complicates centralized ATM.

A partially decentralized ATM architecture is presented in [92]. The authors believe that a totally centralized solution is computationally extremely complex and expensive, while a fully decentralized ATM would be unacceptable. Thus the paper proposes a trade off, introducing a middle ground solution. "Smart aircraft" are introduced, equipped with a Flight Management System with the ability to compute route changes and to perform conflict detection and resolution. Concurrently, a central authority gets control in highly congested airspace.

Another partially decentralized system is proposed in [2]. Here, each aircraft is given an initial schedule defined by a sequence of points specifying its route from start to end. If any problem arises, an aircraft has the possibility to locally modify its trajectory solving an optimization problem. The proposed method considers a set of agents (i.e., aircraft), each one aware of the state and goal of every agent in a predefined region. Therefore, at every time step, each agent has to solve an optimization problem requiring information on its neighbours' position and goal details. Each agent then uses the computed solution for the subsequent movements. Note that, for large enough regions, the problem comes down to classic centralized management.

A novel concept named "free flight" [35] paves the way toward decentralization. It defines a system in which aircraft do not have predefined routes. Free flight gives the possibility to partially or completely eliminate centralized ATM, giving the entire power of choosing route, speed and altitude to aircraft themselves. One key aspect when dealing with decentralized control systems is defining ways to identify and solve conflicts: to avoid collisions, free flight uses the idea of an imaginary area surrounding each aircraft, called protected zone, where no other aircraft is allowed; surrounding such zone is the alert zone: if a generic aircraft is in the alert zone of another aircraft, both have to start a protocol of conflict resolution. Several conflict resolution approaches have been proposed over the last two decades, relaying on pilots or on automated conflict resolution algorithms.

In particular, [56] proposes free flight conflict resolution strategies, analysing it both as a single and as a multi-objective optimization problem. In the former case, the objective function is the sum of flight times of each aircraft and gas mileage, and constraints are defined by aircraft technical requirements (e.g., maximum and minimum altitude). In the latter case, the function to be optimized is a vector which represents the fact that the considered aircraft may be motivated by several goals (e.g., delay, gas consumption).

Another research work on conflict resolution is presented in [91] where the authors describe two possible strategies, named non-cooperative and cooperative, and propose several conflict resolution methods using different techniques. Noncooperative conflict resolution usually takes place in emergency situations when aircraft do not share any information and solve conflicts with no coordination at all. The cooperative one describes situations where aircraft jointly decide the strategy to be applied. Note that, in non-cooperative conflict resolution, aircraft have a finite number of collectively feasible behaviours and have to select a subset of them which prevents any possible arising conflict.

A further decentralized conflict resolution method is proposed in [22]. Conflicts are handled from the aircraft point of view with the aim of developing decoupled conflict resolution procedures: each local conflict resolution procedure is independent from the ones applied in different locations. The paper first defines necessary and sufficient conditions for the existence of such procedures. Then, it analyses several possible conflicts which meet these conditions and may therefore be solved through the proposed procedures.

One more conflict resolution approach is described in [68]: the paper proposes a negotiation process for decentralized conflict resolution based on game theory. Each aircraft is seen as a player and conflict resolution is tackled as a two-player game where each player aims to find a route minimizing a predefined cost function. An interesting feature of this method is the fact that, during negotiation, each aircraft has to reveal its proposed strategies to solve the conflict, but it does not have to give any other information (e.g., cost function).

In [53], an approach is proposed with aircraft considered as autonomous hybrid automata, i.e., time invariant hybrid systems without inputs. Specifically, the paper analyses three possible cases where one, two or three aircraft are considered. In particular, given a set of predefined aircraft, it defines possible evolutions for their state. It analyses all possible trajectories and computes the optimum one, preventing any type of possible collision. When two or more aircraft are considered, the computed optimal solution, known as Roundabout hypothesis, is for aircraft to coordinate their trajectory as classic car movements at roundabouts.

A MAS approach is presented in [6] with agents (i.e., aircraft) moving in a network described as a graph in which nodes are unique way-points. Agents iteratively communicate with each other by sending messages containing logistic information (e.g., location, altitude speed). Each agent has to perform a three-step decision process: a perception step, i.e., 
agents receive and store information about neighbour agents; a decision step, i.e., based on the received information, agents upload their velocity to avoid possible collisions; an action step, i.e., agents update their information message and send it to other agents.

This analysis shows that in decentralized air traffic management two main issues emerge: trajectory optimization and conflict detection and resolution. Although in principle the same holds for real-time railway traffic management, a major difference exists. Specifically, the degree of freedom of aircraft as for trajectory is much higher than the one of trains, which have predefined intermediate stops and have anyway to travel on existing tracks. It is true that typically aircraft travel on airways, i.e., on specific routes in the airspace, but airways aim is mostly the simplification of air traffic controllers' tasks. In free flight, no constraints need to be imposed and traffic management approaches are designed consequently. Therefore, when dealing with air traffic management, conflict resolution techniques can exploit a significantly higher flexibility. On the opposite, fewer speed profile possibilities are available for aircraft: while a train can stop and wait for virtually any time lapse, aircraft always need to keep a minimum safety speed. This makes air traffic and railway traffic management greatly unequal with respect to essential problem characteristics. Hence, the proposals summarized in this section are not directly exportable to the problem we consider in this paper.

\section{Waterway Traffic System}

As air and railway traffic, waterway traffic is currently managed in a centralized way by the Vessel Traffic Service (VTS): a service or set of services set up by competent authorities, designed to increase the safety and efficiency of waterway traffic and to protect the marine environment. Recently, several works have been proposed for the study of vessel motions and conflict detection.

On the first topic, [95] and [96] make a big use of data provided by the Automatic Identification System (AIS). This is an automatic tracking system used in the naval field, which includes information about identification number, speed, route and position of vessels. In particular, both papers use AIS statistics as input and model validation data for the proposed MAS. The simulations turn out to develop realistic situations which may be useful for many goals, e.g., to simulate the construction of structural works, to analyse the occurrence of possible conflicts, to develop more efficient traffic methods. Although it is not the focus of these papers, they propose decentralized approaches thanks to which vessels autonomously navigate in the waterway network.

Always using AIS data, [97] proposes a three-step MAS method for waterway traffic management. The first one is a learning step, where AIS data is used as basis for vessel motions and how they relate with each other. The second step requires sensing, i.e., forecast information on the surroundings, to enable better navigation. The third step is the planning one: it consists of programming route schedules. Several types of agents interact with each other, including vessel agents that simulate vessels behaviour, and shore station agents that handle long-range route scheduling.
Similarly, [84] proposes a MAS approach: maritime space is split into a variety of zones organized as nodes of a directed acyclic graph. Waterway traffic management is carried out by traffic control agents, and vessels are represented by individual agents. Historical data are used to generate and validate the simulator.

A further approach using agents is presented in [87]. The paper tackles the problem of vessel movements and interactions using a multi-agent path finding method: an iterative algorithm computing vessel paths is proposed, and it uses AIS data as an input. Such algorithm works either in historical mode, i.e., using historical AIS data, or in live mode, working with realtime streams of information. At each iteration, a coordination path finding procedure is performed to set up coordinated paths for neighbouring agents. A proof of the proper functioning of this algorithm is proposed by the same authors in [88].

An interesting study aiming to mimic human decision making is proposed in [86]. The paper develops a model to handle vessel motions, as well as to manage possible dangerous events. The model is based on the military strategy named Observe Orient Decide Act (OODA). Conceptual Blending Theory, i.e., a theory explaining the human procedure for dealing with information, is used to model OODA to develop a working thread assessment model. By doing so, the paper implements a human behavioural simulation model to process vessel movements and thread assessments.

A distributed problem solving network as an approach for waterways traffic management is proposed in [42]. Vessels are all processing nodes in the proposed network. They share information thanks to a communication protocol. The paper elaborates organizational structuring and an expectation-based negotiation mechanism to get to effective vessels cooperation.

To achieve a distributed waterway traffic management, [43] suggests a cooperation method mainly based on inter-vessels communication. The paper proposes a five-step cooperative manoeuvring procedure in a secure and well-functioning conflict resolution approach. When a conflict is detected, vessels communicate with each other and make out local conflict resolution plans. To make sure that feasible local plans exist, hierarchical structures are created, so that global decisions are made based on needs of most "important" vessels.

In the same way as for air traffic management, decentralized waterway traffic management has a prominent degree of freedom characterizing routes and conflict resolution which is not to be found in railway networks. As for air traffic, the main difference between railway and waterway systems lies in their basic structure: the first is characterized by predefined and fixed paths constrained by tracks, and they cannot be changed no matter how critic a situation is. The latter can exploit more flexible routes (sea lanes) with the actual possibility of being modified when needed. Differently from the air traffic management case, in waterway complete stops can be envisaged, although they are not as simply achieved as in railway. Therefore, as above, research works dealing with waterway traffic management can hardly be exploited for the problem we are tackling. 


\section{Modelling Possibilities for Decentralized REAL-Time RaILWAY TRAFFiC Management}

As discussed in Section IV, the literature on other modes of transport cannot be easily exported to real-time railway traffic management. Hence, in this section we analyse different approaches which may be at the basis of the emergence of an effective behaviour of a decentralised system as the one we are considering in this paper. Recall that trains' behaviour concretises in having each train taking one of the routes available for performing its mission and respecting precedences at junctions when it needs to let another train pass first. This behaviour brings to a collective system's behaviour, which can be assessed considering a function of resulting delays. The approaches we present in this section are of two types.

On the one hand, we present approaches in which the behaviour of the system emerges from the application of predefined rules without any actual decision made by trains. Rules may come from some hierarchy relations between trains (Section V-A) or be driven by task allocation principles, where a task may be seen as passing first at a junction (Section V-B). Moreover, they may be automatically learned from the historical outcomes of similar situations (Section V-C).

On the other hand, we describe approaches in which the behaviour of the system results from trains decisions, on which they reach an agreement. This agreement may be reached thanks to the attainment of a consensus (Section V-D) or to an auction negotiation (Section V-E). Finally, we analyse the case of system components that simultaneously cooperate and compete for making the best decisions (Section V-F).

\section{A. Hierarchy}

A hierarchy is the organization of components or individuals into groups, based on their degree of importance. The development of hierarchical systems is very common in social structures of any kind. In these systems, the higher the importance of a member is, the more it has privileges and power. Dominance hierarchy formations are a common peculiarity of animal kingdoms, where animals behave based on their hierarchical ranking. The accepted explanation for the presence of animal hierarchies is the so called winner-loser effect. With winner-loser effect it is defined the condition in which the individual possibility of winning or losing a contest is affected by previous results. Namely, if an individual wins a contest, this raises its probability of winning the following one; respectively, with the losing effect, losing a contest decreases the probability of winning in the future.

Modelling a hierarchical structure in the problem of decentralized real-time railway traffic management may be considered as defining a hierarchy of trains. As we will see, this hierarchy may be implied by many factors and may define the order of passage of trains at a junction. Specifically, suppose that a train suffers a delay with respect to the timetable, causing the occurrence of a conflict: if it ran at the planned speed, it would use a junction at the same time as another train. The conflict corresponds to a contest. In this case, the hierarchical ranking between the two trains may be used to identify the winning one, i.e., the one that has the power to decide which one passes first.

Winner-loser effects have been widely discussed in animal groups. In [26] a model for the formation of hierarchies is proposed. Each individual is characterized by two factors: the winner-loser effect and the resource holding power (RHP). The first one, as already explained, is strictly related to the results of the previous contests; instead the RHP is a physical attribute that defines the capability of an individual of winning or losing (e.g., its strength). An important feature of the proposed model is that, when a contest occurs, each individual has no information about its opponent except for a time invariant assessment of its RHP. The model is used to examine how winner-loser effects affect the formation of hierarchical structures. It considers different cases: when only one between the winner and the loser effect is significant and if both winnerloser effects matter. The fact of having no information on the opponents is no longer present in [27], where the same research group presents an updated version of the model. Each individual has an estimate of its opponent's RHP. The accuracy of the estimation changes and its effects on the formation of hierarchies are studied.

The two models just mentioned are the central topic of [47]. The paper evaluates and analyses the results of these models on the basis of simulations, mainly focusing on the number of contests necessary for the emergence of hierarchical structures.

A review of previously published works is also proposed in [52]. It analyses [26] together with the models in [3] and [40], both examining the emergence of hierarchies due to winner-loser effects. Apart from examining the accuracy of these models to represent the observed behaviour of groups of individuals, an interesting aim of the review is to try finding alternative ways of defining the RHP. As already said before, the RHP is an inner value that specifies the strength of each individual and, therefore, the models do not give a mathematical formulation to quantify it. The paper links the RHP of an individual to the number of times it won and lost, making it easy to compute.

The idea proposed by these models could fit well our goal of modelling decentralized real-time railway traffic management, at least in a first step. Both winner-loser effect and RHP value are adaptable concepts in the case of railway. As mentioned above, a contest takes place when more than a train wishes to cross a junction at the same time or in a short interval. It is trivial to see that if only two trains are meeting, winning a contest is equivalent to going first. Instead, if several trains meet, it is less immediate to understand who wins and who loses. A way of dealing with more trains could be to rank the ones having to cross a junction and assign a value to each train based on its position on the obtained list. The winner-loser effect can be seen as based on the history of each train, defined by what happened in the previous meetings. In a simple design, the RHP value could represent the different types of trains that may have different priority, e.g, high-speed trains, conventional trains, freight trains and dangerous freight trains. Note that the use of this kind of model makes the background information and the predefined priority of each train affect its path, but does not consider any other factor 
which may have an impact on the importance for a train to go first.

Somehow allowing the consideration of other factors, a different approach is proposed in [7], where hierarchy formations are established using game theory. In particular, this work uses a multi-player version of the Hawk-Dove game. In this game, when two players aim to the control of a resource, they can chose between an aggressive hawk strategy or a passive dove one. The choice of the strategy depends on the expected reward obtainable with it. When two hawks fight, they go on until one decides to leave because of the injuries sustained; if two doves meet, they don't fight but equally share the resource; when a dove and a hawk meet, the dove leaves and the hawk gets the entire resource. Hence, this model has the peculiarity of not having past results interfering with the possibility of winning or losing a contest: no winning-losing effect is present but, based on the strategy chosen, an individual has a defined probability of winning. An earlier and more elaborated version was proposed by the same research group in [8], where more than two individuals can compete at the same time. Although this version may be suitable to represent many situations, it comes with a significant increase of complexity. This complexity is not always acceptable, especially in dynamic environments. The idea of using strategies as proposed in [7] and [8] could be interesting for the problem we are considering, moving a step toward train decision making. To apply it, it would be necessary to define what the reward associated to a strategy is in the trivial case of several trains meeting at a junction: these rewards may be linked to the features of the trains but also to external factors such as delay, number of passengers or importance of the goods transported. As a further step, this model could be extended to a more complex system, with the consequence of having to deal with multiple agents and a large set of all possible strategies, for example based on the need to cross several junctions one after the other. Moreover, the possibilities of having or not perfect information on other agents rewards would be an interesting development, particularly suitable for the railway traffic context in which we may think of different railway undertakings not wanting to share their utility functions. As mentioned above, unlike the winner-loser approaches, this model can value how important it is for a train to go first. In this way, the model allows to better deal with peculiar situations, for example when two trains meet right after the departure, and hence without any background on previous contests.

\section{B. Task Allocation}

The problem of assigning tasks to a collection of agents is known as task allocation and is a very common problem in everyday life applications. A remarkable example is easily recognizable in all the most famous electronic commerce companies, such as Amazon or eBay, funded in the last couple of decades. In fact, when an order arrives, it is assigned to an agent, usually a robot, that takes care of the preparation tasks. These companies usually have to deal with a huge number of shipments per day, making the process of organizing the distribution of tasks to different agents essential.
In real-time railway traffic management, assigning tasks to a set of trains needing to cross a junction may mean deciding the order of the trains: the tasks may be going first, second and so on. Task allocation rules may then define train precedences. Alternatively, from a dual perspective, a junction may be the focus of the model and tasks may correspond to the fact of being traversed by each train. Allocating tasks in a specific order would bring to define trains' precedence. Indeed, in a complex network including several junctions and the possibility of re-routing, these examples cannot be so easily applied. However, it may be possible to think of a mechanism that allocate tasks considering their interdependence.

The definition of a taxonomy of task allocation problems is the central topic of [37] and [59]. In [37], multi-robot task allocation problems are classified using three features: robot, task and time. Different approaches are proposed if: robots can manage only one task at a time or are capable of handling more tasks; tasks require one or more robots; allocation time must be immediate or can be planned in advance. Similarly, [59] classifies task allocation problems but focusing on temporal and ordering constrains. Hence, tasks are classified based on their urgency of being carried out and on the specific order they need to be executed in. In [37], all the approaches considered are performed in a centralized way. Instad, [59] gives a brief categorization of decentralized approaches underlining, however, the limited number of existing works.

In [31] the problem of task allocation is analysed in the context of MAS, proposing a model called TRACE (Task and Resource Allocation in Computational Economy). The paper characterizes each task by four factors: type, duration, deadline and priority. The type factor specifies the necessary characteristics that an agent should have in order to accomplish the task; the amount of time needed to complete the task is the duration; the deadline gives information about the latest possible time for a task to be carried out; the priority tells how important the task is. Each agent to whom a task can be allocated, can be different and have dissimilar abilities from the others. This matching between task and agent's capability to perform it, somehow leads back to the ideas of hierarchies: the most suitable agent wins the task. However, a more elaborated procedure is proposed here to take into account of the multiplicity of capable agents and of their organization. Specifically, the TRACE model groups agents in so-called organizations. In each of them there is an agent in charge or resource management and a set of agents who may be either permanent or marketable. A task can be allocated either to an agent or to an organization. When it is allocated to an agent, the resource manager can ask for help to the other agents in the organization. When it is assigned to an organization, the resource manager decides if it can be accomplished with the available resources. Otherwise, he can ask for extra help to the other organizations who may accept to temporarily share their marketable agents. In our opinion, this model is not appropriate for modelling real-time railway traffic management, due, first of all, to the very specific characteristics that define each task (i.e., type, duration, deadline and priority). In the problem we are interested in, tasks characteristics would strictly depend on the train-agent performing them. For example, the task 
"going first through a junction" may have different durations depending on the selected train-agent. If it is allocated to a high-speed train running at full speed, it will have a very short duration. If it is allocated to a freight train right after a stop, the duration will be much longer. Also, if thinking to the dual perspective of a representation focused on junctions, the same issue remains: if the order of tasks allocated to a junction is high-speed train first and freight train second, the task durations will be different from the case of opposite ordering. Specifically, if the "high-speed train" task is performed first, it will have the duration corresponding to a free network run. Instead, if it is performed second, additional braking and acceleration times will have to be included in the task duration. The same holds for the "freight train" task, although the specific times will be different from those of the high speed train. A further aspect which makes the TRACE model unsuitable for our problem is the idea of organizations possibly sharing tasks' burden among different agents which is not really compatible with the definition of trains precedences. Differently, the concept of grouping agents in organization which manage tasks internally may be an interesting hint to be exploited. In particular, it may represent the case of several trains having to cross a junction and belonging to few railway undertakings. Here, the task "going first through a junction" may be allocated to a railway undertaking-organization, which would decide to which of its trains-agents assign it.

A mathematical approach to the task allocation problem is presented in [30]. The paper studies the problem of allocating tasks to a set of agents represented as nodes in a graph: two nodes are linked if the corresponding agents can communicate with each other. The authors propose a distributed iterative algorithm to perform a task allocation through a linear programming model. At each iteration a subset of tasks are allocated to agents in the neighbourhood of a randomly selected one. If throughout successive iterations all tasks can be allocated, the algorithm stops. Otherwise some constrains in the local allocations are relaxed.

In [36] the task allocation problem is studied in the field of a multi-robot system using an Artificial Immune Network model. In the system, B cells produce antibodies that interact with antigens, with the feature that each antibody can interact only with one specific type of antigen. In the paper, robots behave as B cells and tasks as antigens. When a task has to be allocated, each robot is associated to a specific value that describes its capability to perform the task. The proposed algorithm chooses the robot for a specific task based on this value.

The last two models proposed could both represent interesting solutions for modelling decentralized real-time railway traffic management. In particular, the global solution presented in [36], can be almost directly used in our case. The cells allocated to each antigen may be the trains which have to cross a specific junction. The value describing the capability to perform a task may reflect aspects as trains priorities, current state and possibly the cost of the delay which may come from the task allocation. However, capturing aspects as the multiplicity of trains operated by the same railway undertaking or the interdependence of junctions may not be straightforward. Indeed, the former may possibly enter in the capability quantification, while for the latter a different variant of the model needs to be designed.

For applying [30] to our problem, the model proposed should be adapted even for the independent junction case. However, it is particularly interesting due to the use of the concept of neighbourhood. In our case, the neighbourhood may be interpreted in temporal terms: the task allocation may be done only considering the trains which get to a junction in a certain time interval. As time passes, the interval may shift and, hence, the neighbourhood may change. Indeed, such procedure would require the definition of further rules, to replace the centralized management with a decentralized approach in which each train simply follows predetermined rules.

\section{Reinforcement Learning}

An approach quite often used for managing decentralized systems is reinforcement learning. The Swiss Federal Railways (SBB-CFF-FFS) is currently developing such an approach for decentralized real-time railway traffic management [28], although no details on this development are available. Generally speaking, in reinforcement learning the system is in charge of defining the rules which will drive its behaviour. It does so through replicating the same process a large number of times, each time receiving feedback on the quality of the rules applied. Eventually, good rules emerge without the definition of specific criteria: these criteria emerge in the replications and do not need to be fully explained or understood.

In decentralized real-time railway traffic management, this may mean, for example, exploiting the records of historical decisions made by dispatchers in the practice, to learn how to replicate their principles. Alternative to historical data, another option may be to simulate a large number of perturbed traffic situations managed through some optimization algorithm. In principle, any source of data that allows the assessment of good traffic management rules may be the basis of a reinforcement learning approach for our problem.

In [73] and some following papers (e.g., [100]), reinforcement learning is used to schedule jobs on a set of machines in a decentralized decision making framework. The aim is obtaining good scheduling solutions extremely quickly. The vision is resource-based: machines choose in which order they shall complete jobs. Here, learning means iteratively improving the decision policy with respect to the optimization of the global costs. To do so, particular attention needs to be paid to the definition of each resource's choice criterion, which shall reflect as much as possible the global aim. In a railway perspective, this would translate into having a track which needs to be used by several trains to choose which train shall cross it first. The choice should be based on a criterion coherent with the overall system aim, for example delay minimization. This perspective may become unsuitable when one of the possible choices consists in deciding whether to use a track or to re-route.

The opposite, job-centric perspective is very seldom adopted in scheduling approaches. Indeed, it is the choice made by 
SBB-CFF-FFS. Here, trains learn how to define interaction strategies which allow all of them to reach their final station effectively.

Indeed, the idea of using reinforcement learning is valuable if it is possible to define a priori what a good solution is. Long tests are necessary to train the system, and for each of these test it must be possible to state if the strategy chosen brought a good result or not. In a sense, the decentralization through reinforcement learning can be successful to obtain a computationally efficient approach to reproduce the results of a centralized decision. Instead, if the decentralization is aimed at, for example, allowing the non-disclosure of private information, it may not be possible to define a suitable testbed to train the system. In the context of real-time railway traffic management, this non-disclosure condition is indeed an appealing trait of a decentralized system. For example, railway undertaking may prefer not to share information on the internally defined priority of some trains, especially in the context of increasing competition which is envisaged for the system at the European level. Another issue that may hinder the use of an approach based on reinforcement learning in the railway context is the natural lack of understanding behind specific choices. In a competitive system in which actors acceptance is important, it will be in our eyes crucial to be able to explain the functioning of the system. Indeed, this is not an issue in countries as Swiss, where the main railway companies operate their own networks as they run the trains along it. Instead, in countries subject to EU directives, competition is expected to increase in the future.

\section{Consensus}

The decision making process in decentralized systems is often modelled as the consensus problem. Consensus means getting all the components of a system to reach a common decision about certain tasks of interest.

In MAS, reaching consensus consists in a procedure where all the agents in the network interact with each other, with the purpose of reaching an agreement on a common behaviour. The process that leads to consensus is modelled through iterative interaction algorithms where, at each iteration, agents reveal their opinion to the others, moving toward a common behaviour.

In real-time railway traffic management, reaching a consensus may be the process of getting a common decision that all the components of the system need to observe and follow. Specifically, the consensus should be reached on how to use limited resources, i.e., in what order trains should pass through a junction in case of conflict, or what route should each train follow.

Over the last decades the increasingly frequent use of distributed systems has made the design of converging consensus algorithms a central research topic. Overviews focusing on recent works on consensus and MAS are given in the surveys [12] and [62]. Both studies analyse consensus in complex dynamic systems, taking into account the effects of time delay in the general performance.

The problem of consensus under time delays is also studied in [90]. Two different types of time delay are considered: input delay and communication delay. The first defines the delay given by the necessary time for the input to arrive at the agents, the latter is given by the time gap between transmitting information and receiving it. The paper analyses the consensus problem with input delay only and when both information and input delay are present.

A particular kind of consensus problem in MAS is the one that considers one agent as special, with dynamics independent of the others, and is known as leader-following consensus problem. For example, [58] considers the leader following consensus problem in the case of time-varying interaction topology, i.e, when the structure of the interconnection between agents is not fixed but changes over time.

Similarly, [72] considers topologies that change in a dynamic way. It examines the conditions that guarantee the convergence in time-varying networks when the information exchange is not complete and trusted.

The cited works tackle the continuous consensus problem. Continuous consensus decisions require the group to achieve consensus for one of an infinite number of options, while the discrete consensus decisions require the group to choose from a finite set of options. The latter is often referred to as the best-of-n problem, where $\mathrm{n}$ stands for the number of available options. [94] proposes a literature review of the state of the art on the formalization and solution of this problem. For example, [71] considers a population of agents, in which each agent is either committed to one of the available options or is uncommitted. Agents can obtain a noisy estimate of the quality associated to each option. At macroscopic level, a decision is taken as soon as the entire population or a large fraction of it is committed to a single option. At microscopic level, the paper represents the behaviour of individual agents as a probabilistic finite state machine that describes the commitment dynamics. An agent probabilistically and periodically changes between a committed and non-committed state according to two types of transitions: spontaneous and interactive. Spontaneous transitions model the discovery of an option or the abandonment of commitment to it with a given probability. Interactive transitions model the recruitment and cross-inhibition processes resulting from the interaction between differently committed agents.

An important feature that generally characterizes consensus algorithms is that, in order to reach an agreement, they perform a number of iterations in which agents observe each other's behaviour before converging. For example, it may be necessary to reach a consensus on the speed of several agents sharing an infrastructure. Initially all agents travel at different speeds. Throughout iterations, they progressively start moving uniformly, converging to a somehow average speed, or to the speed of the leader. This problem can be relevant in the railway system for synchronizing the speed of trains which follow each other, for allowing energy efficiently and avoiding continuous braking and acceleration mostly in high speed lines [50]. Instead, the definition of trains routes and precedences in realtime railway traffic management cannot be effectively dealt with through iterative adjustments aiming to converge to the same behaviour of all trains. Hence, the algorithms proposed to tackle the consensus problem are not suitable. 
Furthermore, although at first sight discrete consensus problem may closely match real-time railway traffic management, a relevant difference exists. In particular, in discrete consensus, agents do not have individual competing preferences, and they all aim to the best solution for the group. Indeed, it would be socially very good if this could be reproduced in railway, but we do not believe it to be an actual possibility in an increasingly competitive context as the one we consider.

\section{E. Auctions}

Another method that allows coming to an agreement on the use of resources by several agents is represented by auctions. In auctions, several agents bid on one or more resources and the ones that mostly value the resources obtains them. This is the principle behind auctions, and several mechanisms exist to try to enforce it.

In real-time railway traffic management problem, resources may represent the right of passing through a junction at a certain time, or in a certain position of the schedule. Trains would then bid, possibly each valuing the resource in different ways, and the negotiation in the auction would bring to the final routes and precedences. Specific bids would be the result of trains' decision making on the value to assign to each resource. If the auction is designed in particular ways, for example considering bundles of resources rather than single ones, each train's evaluation may remain to some extent private information.

Three main properties that may be sought for in the definition of these mechanisms are budget balance, individual rationality and incentive compatibility [46]. A mechanism is budget balanced when the overall amount paid and received by the agents participating in the exchanges sums to zero. A mechanism is individual rational when no agent is disadvantaged by participating, i.e., the amount paid plus the utility it associates to the acquired resources is always at most equal to the maximum cost or disutility it is ready to bear. A mechanism is incentive compatible if no agent has an advantage from cheating. In general, it corresponds to no agent having an advantage from declaring a willingness to pay different from the true one for a resource. Unfortunately, a mechanism cannot be at the same time budget balanced, individual rational and incentive compatible, as shown in [57]. Hence, at most two of these properties must be privileged in the design of a mechanism.

Auctions have been used in very different contexts. In the transportation field, they are frequently considered to solve pricing problems, for example for the use of automated vehicles [99] or for the definition of highway tolls [85]. In [20], micro-auctions are used to properly set traffic lights in a flow-dependent manner. The idea is to have phases, i.e., specifications of which lanes of traffic at an intersection may flow, bidding for becoming active depending on traffic detections. The phase which proposes the highest bid becomes active (after the appropriate yellow). This approach is not uninteresting from a railway traffic management perspective. However, it may only represent the case of having as objective function the maximization of train flows, regardless the timetable. Even in this case, the flow detection shall be attentively designed, since indeed arrival flows on tracks and roads are very different in magnitude.

Following principles similar to the ones of auctions, [65] proposes a combinatorial exchange mechanism applied to air transport. A combinatorial exchange is an auction mechanism in which several agents negotiate bundles of resources. In the paper, resources correspond to the right for an aircraft of using airport infrastructures or airspace sectors capacity at a given time. Indeed, only times and routes which are coherent with each other are relevant for the exchanges. This problem emerges in the timetable definition phase. Airlines participate to the exchange having an original allocation, which they try to improve. No money exchanges occur, but if an airline desires the use of a highly valued resource, it must be willing to renounce to one or more resources which together bring the same value to the market. A mechanism as the last one may suit the real-time railway traffic management problem quite nicely: railway undertakings may manage several trains which need to cross the network encountering different conflicts. They may be willing to sacrifice a train for favouring another one. Many trains may bid on the same resource, i.e., many trains may be involved in a conflict. Moreover, bundles of resources may be considered: the value associated to a train passing first at a junction also depends on its passing orders at future ones. However, two main issues emerge. On the one hand, a design principle is not straightforward: what happens if a railway undertaking has only one train, and cannot sacrifice anything to obtain good passing orders for it. On the other hand, the size of the problems to tackle may become very large, due to the consideration of all junctions trains may cross. Hence, the computational performance of solution algorithms may hardly be suitable for real-time decision making.

The idea of using auctions to allocate track access rights to railway undertakings has been briefly explored in [81]. Specifically, different existing mechanism are considered, and their merits and lacks are discussed. This work may definitely be used as a basis for defining a decentralized real-time railway traffic management approach, but significant modifications need to be made. In particular, in [81] the timetable definition problem is tackled, which takes place some months before operations. Although this problem is very similar to the one we consider in this paper, in timetabling it is possible that no track is allocated to a train or to a railway undertaking. Instead, in real-time railway traffic management, trains are actually running and they need to be fit in the schedule. To guarantee the accessibility of the network, specific mechanisms may be proposed.

\section{F. Coopetition}

The expression coopetition is the combination of the words cooperation and competition, mainly used in inter-corporations backgrounds. It describes peculiar situations where companies compete in a market but simultaneously make some kind of profit cooperating with each other.

In decentralized real-time railway traffic management, trains can be seen as agents interacting in a coopetition framework. 
They would compete in order to obtain the best conditions for themselves, trying to pass first at junctions and travel along the shortest route. At the same time, though, trains would cooperate for the general performance of the system, with the common aim of getting good results in terms of delays. Hence, their routes and precedences would be fixed taking into account this common aim. This cooperation may bring the indirect reward that emerges in repeated games: each train may have interest in making decisions so that the system is well performing. Although in some cases a train may be penalized by cooperation, the criterion linked to the overall performance indirectly implies that no one is too disadvantaged. Hence, in repetitions of a decision making situation in which a train itself happens to be the most penalized one, it can count on being somehow protected thanks to the system perspective.

The idea of coopetition is often combined in an interesting way with game-theory models where the participants are seen as players of a specific game. In particular, one of the first works to explore the idea of combining coopetition and game theory can be found in [5]. The book proposes to apply the idea of exploiting game theory models in which each agent has more than one possible strategy, analyses the obtainable results and then chooses the best alternative. Specifically, each "player" has to foretell the initial behaviour of its competitors as well as their possible reaction to its moves in order to fully assess all strategies. This work is considered a landmark in the field of business game.

A work that takes inspiration from [5] is [61]. The paper provides a recent review on coopetition and game theory. The paper briefly clarifies why using game theory in the context of coopetition can bring benefits. Moreover, the review presents a mainly generic solution to do so in the context of both normalform and form-based games, i.e., games that are split into sequential independent situations.

As briefly described at the beginning of this section, the idea of coopetion is mainly developed in the field of business, where concrete examples can be found. Specifically, [76] describes the idea of co-branding as some kind of strategic coopetition agreed between two or more companies, specifically between Nike and Apple. After calculating basic data on these companies (e.g., annual growth rate) the paper analyses the possible pay-offs of the coopetition using a decision tree. Results are discussed afterwards, coming to the conclusions that both companies would benefit from a coopetitive alliance.

Somehow in a similar spirit, [60] analyses the problem emerging in the context of Japanese insurance companies using a game theoretical approach. The paper examines the problem using a well-detailed scheme: the suggested model is split into two stages. It considers two companies having to choose how to behave in the market while keeping the behaviour of the other under control. In the first stage of the game each company independently chooses how much of its budget to invest in loss-prevention advertisement, described using an investment cost function. Then, investments are made public and the second phase of the game begins: policy sales investment are chosen and based on these choices the game is analysed looking for a possible equilibrium. An aspect that makes this paper particularly interesting and that is not always present in the literature, is the fact that the whole game is formally described using a mathematical formalization, clarifying the details of the proposal and making its reproduction possible. Indeed, a variant of this formalization may be proposed for the real-time railway traffic management problem. However, the paper analyses the very trivial case when only two participants are considered. The problem we are trying to examine is way more complex in both the number of members and the amount of parameters. Hence, even if this work may be seen as a first step in the right direction, it has to be deeply extended to be actually used for decentralized real-time railway traffic management.

\section{CONCLUSIONS}

In this paper, we proposed an analysis of the state of the art aiming to identify possible ways to model decentralized real-time railway traffic management. This problem consists in defining trains' routes and precedences when perturbations occur and the planned timetable becomes infeasible. The one proposed is a new approach opposed to the currently deployed centralized systems. Although still being virtually unexplored in academic literature, its potential is investigated by railway infrastructure managers in different European countries. Indeed, industry sees that decentralized decision making may allow the development of a system featuring important characteristics which lack in the current centralized one.

We first described the current literature on centralized and decentralized real-time railway traffic management systems. This showed how little has been done concerning the latter. Thus, we focused on the more extensive existing literature on other transport modes, seeking for possibilities of crossfertilization. Namely, we considered road, shared mobility, air and waterways systems. Although intuitively these systems may appear quite similar to the railway one, we realised that important differences exist and this convinced us to head toward other directions.

In particular, we analysed the process which may be followed to reach a common behaviour in decentralized systems. Specifically, we focused on rule-based processes, in the form of hierarchies, task allocation or reinforcement learning, and on decision making ones, modelled as consensus seeking, auctions and coopetition. For most of these processes, we found and pointed out promising ideas that could be actually used for modelling decentralized real-time railway traffic management.

After this analysis, we think that a rule-based approach may be used as a starting point. These approaches have the advantage of translating in quite simple algorithms, which may thus find solutions to the trains' routes and precedences definition problem in very short times. Moreover, their simplicity may ease their explanation to the community. Indeed, although part of the industry is already looking with interest at decentralization, there is still a long way to go before it is considered as actually possible for deployment. In this perspective, we think a hierarchical approach could be the most appropriate, although task allocation ones are also promising. As for learning approaches, despite their undeniable interesting characteristics, we think their utilization is made more complex by the need of very big databases necessary for the learning itself. 
While using simple approaches to widen the acceptance of decentralized real-time railway traffic management, we think academia may focus on more complex ones for enhancing the performance of the system. Indeed, the system must be capable of achieving performance that are comparable with those of the current state of the art on centralized approaches. We doubt that rule-based approaches may be able to do so. Instead, decision making ones may definitely succeed. Namely, we think that auction-based approaches look the most promising, although significant effort must still be done for making them applicable to the problem at hand. Probably, also coopetition approaches may be explored, but their definition is quite light for the moment and the effort required may hence be even bigger.

In future research, we will work on the actual instantiation of some of the identified promising ideas. This will most likely allow a further shrink of the panel of possibilities, up to the identification of a single most promising one to be developed and deeply studied.

A decentralized approach may allow overcoming some important issues of the current system. Once such an approach is formalized and tested, the choice between decentralized and centralized system shall be considered as a trade-off. This trade-off is nicely presented in [80], as a sort of key for the analysis of current practice in different European countries. In the paper, decentralization is interpreted differently from what we do here. We intend it as having the system behaviour emerging from the combination of individual trains behaviour. It may be rule-based or result from an actual decision making process, but in any case it does not involve any organization having decisional power over all trains in an area. In [80], a system is decentralized when local control centres with decisional autonomy exist. Despite this different interpretation, the analysis made in the paper fits our scope, too. Specifically, deciding whether let a centralized organization make decisions or decentralize them requires a very good understanding of the system to anticipate how it may be affected by losses and benefits. For example, a centralized approach warrants a general and global perspective, hence facilitating rapid and decisive coordinated actions. Instead, a decentralized system may exploit the high flexibility brought by a very detailed knowledge of the local context and a direct control over resources.

\section{ACKNOWLEDGEMENTS}

The work of Elisa Marcelli was supported by University of Camerino, Italy, with a six-month scholarship for recent M.Sc graduated for further study or training outside Italy. The authors would like to thank Professor Renato De Leone who made the collaboration between University of Camerino and IFSTTAR possible.

The work of Paola Pellegrini was partially funded by the CPER ELSAT2020 project co-financed by the European Union with the European Regional Development Fund, the French state and the Hauts-de-France Region Council.

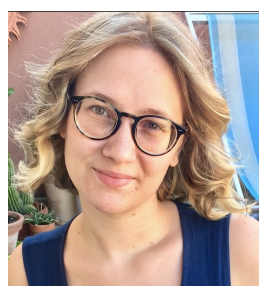

Elisa Marcelli received the M.S. degree in Mathematics and Applications from the University of Camerino, Camerino, Italy, in 2017. She is currently working towards the Ph.D. degree in Mathematics at the University of Camerino. She has been a Visiting Researcher at the French institute of science and technology for transport, development and networks (IFSTTAR), Université Lille Nord de France, Lille, France, for a semester in 2018 and at Carnegie Mellon University, Machine Learning department, Pittsburgh, USA, in 2019. Her research interests focus on Data Science. In particular, she is interested in the projection of data sets into high-dimensional spaces.

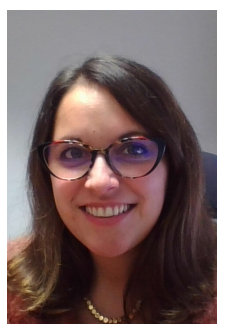

Paola Pellegrini received the Ph.D. degree in Mathematics for Economics from Ca' Foscari University, Venice, Italy, in 2007. She is currently research director at the French institute of science and technology for transport, development and networks (IFSTTAR), Université Lille Nord de France, Lille, France. She has been a visiting researcher at the University of Arizona (USA) and she has worked as researcher at the University of Trieste and Ca' Foscari University (Italy), and at the research institute IRIDIACoDE (Belgium). She has participated in research projects in the field of mathematical optimization, air traffic management, and railway transportation. Particularly, she works on optimization methods for the exploitation of capacity in transport network, with particular attention to realtime railway traffic management. She has published the results of her research studies in several scientific journal articles, book chapters, and conference papers. 


\section{REFERENCES}

[1] L.D. Baskar, B. De Schutter, and H. Hellendoorn. Decentralized traffic control and managemnt with intelligent vehicles. In Proceedings of the 9th TRAIL Congress 2006, 2006. 16 pages.

[2] A. Bicchi, A. Marigo, G. Pappas, M. Pardini, G. Parlangeli, C. Tomlin, and S.S. Sastry. Decentralized air traffic management systems: Performance and fault tolerance. IFAC Proceedings Volumes, 31(27):259 264, 1998. IFAC Workshop on Motion Control (MC'98), Grenoble, France, 21-23 September.

[3] Eric Bonabeau, Guy Theraulaz, and Jean-Louis Deneubourg. Dominance orders in animal societies: the self-organization hypothesis revisited. Bulletin of mathematical biology, 61(4):727-757, 1999.

[4] Emmanuel Bougna and Yves Crozet. Towards a liberalised european rail transport: Analysing and modelling the impact of competition on productive efficiency. Research in Transportation Economics, 59:358 - 367, 2016.

[5] Adam M Brandenburger. Co-opetition. Crown Business, 1998.

[6] R. Breil, D. Delahaye, L. Lapasset, and E. Féron. Multi-agent systems to help managing air traffic structure. Journal of Aerospace Operations, 5(1-2):119-148, 2017.

[7] M Broom and C Cannings. Modelling dominance hierarchy formation as a multi-player game. Journal of theoretical Biology, 219(3):397413, 2002

[8] M Broom, C Cannings, and GT Vickers. Multi-player matrix games. Bulletin of mathematical biology, 59(5):931-952, 1997.

[9] Birgit Burmeister, Afsaneh Haddadi, and Guido Matylis. Application of multi-agent systems in traffic and transportation. IEE ProceedingsSoftware, 144(1):51-60, 1997.

[10] C4R Consortium. Recommendations for a european standard for traffic management under large disruptions. Deliverable 3.3.2 of the Capacity4Rail - Towards an affordable, resilien, innovative and highcapacity European Railway System for 2030/2050. Project funded by the European Union's Seventh Framework Programme, 2017.

[11] V. Cacchiani, D. Huisman, M. Kidd, L. Kroon, P. Toth, L. Veelenturf, and J. Wagenaar. An overview of recovery models and algorithms for real-time railway rescheduling. Transportation Research Part B: Methodological, 63:15-37, 2014.

[12] Yongcan Cao, Wenwu Yu, Wei Ren, and Guanrong Chen. An overview of recent progress in the study of distributed multi-agent coordination. IEEE Transactions on Industrial informatics, 9(1):427-438, 2013.

[13] N.M. Carter and J.B. Cullen. A comparison of centralization/decentralization of decision making concepts and measures. Journal of Management, 10(2):259-268, 1984.

[14] R. Claes, T. Holvoet, and D. Weyns. A decentralized approach for anticipatory vehicle routing using delegate multiagent systems. IEEE Transactions on Intelligent Transportation Systems, 12(2):364-373, 2011.

[15] F. Corman, A. D'Ariano, D. Pacciarelli, and M. Pranzo. A tabu search algorithm for rerouting trains during rail operations. Transportation Research Part B: Methodological, 44:175-192, 2010.

[16] F. Corman and L. Meng. A review of online dynamic models and algorithms for railway traffic management. Intelligent Transportation Systems, IEEE Transactions on, 16(3):1274-1284, 2015.

[17] Francesco Corman, Andrea D'Ariano, Dario Pacciarelli, and Marco Pranzo. Dispatching and coordination in multi-area railway traffic management. Computers \& Operations Research, 44:146-160, 2014.

[18] Francesco Corman, Andrea DAriano, Dario Pacciarelli, and Marco Pranzo. Evaluation of green wave policy in real-time railway traffic management. Transportation Research Part C: Emerging Technologies, 17(6):607 - 616, 2009.

[19] Francesco Corman, Andrea DAriano, Dario Pacciarelli, and Marco Pranzo. Centralized versus distributed systems to reschedule trains in two dispatching areas. Public Transport, 2(3):219-247, 2010.

[20] M. Covell, S. Baluja, and R. Sukthankar. Micro-auction-based trafficlight control: Responsive, local decision making. In 2015 IEEE 18th International Conference on Intelligent Transportation Systems, pages 558-565, 2015.

[21] A. D'Ariano, F. Corman, D. Pacciarelli, and M. Pranzo. Reordering and local rerouting strategies to manage train traffic in real-time. Transportation Science, 42(4):405-419, 2008.

[22] S. Devasia, D. Iamratanakul, G. Chatterji, and G. Meyer. Decoupled conflict-resolution procedures for decentralized air traffic control. In 2009 IEEE Control Applications, (CCA) Intelligent Control, (ISIC), pages $837-842,2009$.
[23] A Chaali Djelassi, Philippe Polet, and Frédéric Vanderhaegen. A comparative study between subjective and objective results of a predicting human behaviour method based on the expected utility and the iterative learning control. application to the car driving. In Systems, Man and Cybernetics, 2006. SMC'06. IEEE International Conference on, volume 5, pages 4360-4367. IEEE, 2006.

[24] Arnaud Doniec, René Mandiau, Sylvain Piechowiak, and Stéphane Espié. A behavioral multi-agent model for road traffic simulation. Engineering Applications of Artificial Intelligence, 21(8):1443-1454, 2008.

[25] Kurt Dresner and Peter Stone. Multiagent traffic management: A reservation-based intersection control mechanism. In Proceedings of the Third International Joint Conference on Autonomous Agents and Multiagent Systems-Volume 2, pages 530-537. IEEE Computer Society, 2004

[26] Lee Alan Dugatkin. Winner and loser effects and the structure of dominance hierarchies. Behavioral Ecology, 8(6):583-587, 1997.

[27] Lee Alan Dugatkin and Aaron David Dugatkin. Extrinsic effects, estimating opponents' rhp, and the structure of dominance hierarchies. Biology letters, 3(6):614-616, 2007.

[28] Adrian Egli and Erik Nygren. Multi-agent reinforcement learning for railways dispatching. https://www.researchgate.net/project/ Multi-Agent-Reinforcement-Learning-for-Railways-Dispatching. Accessed: 2019-02-20.

[29] Patrick AM Ehlert and Leon JM Rothkrantz. Microscopic traffic simulation with reactive driving agents. In Intelligent Transportation Systems, 2001. Proceedings. 2001 IEEE, pages 860-865. IEEE, 2001.

[30] Maria Pia Fanti, Agostino M Mangini, Giovanni Pedroncelli, and Walter Ukovich. Discrete consensus for asynchronous distributed task assignment. In Decision and Control (CDC), 2016 IEEE 55th Conference on, pages 251-255. IEEE, 2016.

[31] S Shaheen Fatima and Michael Wooldridge. Adaptive task resources allocation in multi-agent systems. In Proceedings of the fifth international conference on Autonomous agents, pages 537-544. ACM, 2001.

[32] J Alexander Fax and Richard M Murray. Information flow and cooperative control of vehicle formations. IEEE transactions on automatic control, 49(9):1465-1476, 2004.

[33] Alexander Fay. Decentralized railway control based on autonomous agents. IFAC Proceedings Volumes, 33(9):83 - 88, 2000. 9th IFAC Symposium on Control in Transportation Systems 2000, Braunschweig, Germany, 13-15 June 2000.

[34] Jacques Ferber. Multi-agent systems: an introduction to distributed artificial intelligence, volume 1. Addison-Wesley Reading, 1999.

[35] RTCA (Firm) and Radio Technical Commission for Aeronautics. Task Force 3. Final Report of RTCA Task Force 3: Free Flight Implementation. RTCA, Incorporated, 1995.

[36] Yunyuan Gao and Zhizeng Luo. Dynamic task allocation method based on immune system for cooperative robots. In Intelligent Control and Automation, 2008. WCICA 2008. 7th World Congress on, pages 10151020. IEEE, 2008

[37] Brian P Gerkey and Maja J Matarić. A formal analysis and taxonomy of task allocation in multi-robot systems. The International Journal of Robotics Research, 23(9):939-954, 2004.

[38] Maxime Guériau and Ivana Dusparic. Samod: Shared autonomous mobility-on-demand using decentralized reinforcement learning. In 201821 st International Conference on Intelligent Transportation Systems (ITSC). IEEE, 2018. 6 pages.

[39] Hamid Hassanabadi, Bijan Moaveni, and Mohammad Karimi. A comprehensive distributedarchitecture for railway traffic controlusing multi-agent systems. Proceedings of the Institution of Mechanical Engineers, Part F: Journal of Rail and Rapid Transit, 229(2):109-124, 2013.

[40] Charlotte K Hemelrijk. Towards the integration of social dominance and spatial structure. Animal Behaviour, 59(5):1035-1048, 2000.

[41] Philippe G. Howlett and Peter J. Pudney. Energy-Efficient Train Control. Springer-Verlag, 1995.

[42] Y. Jin, T. Koyama, and Z. . Zhang. The marine traffic control system as a distributed problem solving network. In 1990 IEEE International Conference on Systems, Man, and Cybernetics Conference Proceedings, pages 876-881, 1990.

[43] Yan Jin and Takeo Koyama. On the design of marine traffic control system (2 nd report). Journal of the Society of Naval Architects of Japan, 1988(164):240-252, 1988.

[44] Paul Johnson, Duc Nguyen, and ManWo Ng. Large-scale network partitioning for decentralized traffic management and other transportation applications. Journal of Intelligent Transportation Systems, 20(5):461473, 2016. 
[45] H. Khadilkar. A scalable reinforcement learning algorithm for scheduling railway lines. IEEE Transactions on Intelligent Transportation Systems, 20(2):727-736, 2019.

[46] V. Krishna. Auction Theory. Academic Press, 2nd edition, 2010.

[47] Klodeta Kura, Mark Broom, and Anne Kandler. Modelling dominance hierarchies under winner and loser effects. Bulletin of mathematical biology, 77(6):927-952, 2015.

[48] Leonardo Lamorgese and Carlo Mannino. An exact decomposition approach for the real-time train dispatching problem. Operations Research, 63(1):48-64, 2015.

[49] Leonardo Lamorgese, Carlo Mannino, Dario Pacciarelli, and Johanna Törnquist Krasemann. Train dispatching. In R. Borndörfer, T. Klung, L. Lamorgese, C. Mannino, M. Reuther, and T. Schlechte, editors, Handbook of Optimization in the Railway Industry, number 268 in International Series in Operations Research and Management Science, pages 265-283. Springer New York LLC, 268 edition, 2018.

[50] Shukai Li, Lixing Yang, and Ziyou Gao. Adaptive coordinated control of multiple high-speed trains with input saturation. Nonlinear Dynamics, 83(4):2157-2169, 2016.

[51] Ying Li, Shoufeng Ma, Wu Li, and Huanchen Wang. Microscopic urban traffic simulation with multi-agent system. In Information, Communications and Signal Processing, 2003 and Fourth Pacific Rim Conference on Multimedia. Proceedings of the 2003 Joint Conference of the Fourth International Conference on, volume 3, pages 1835-1839. IEEE, 2003.

[52] W Brent Lindquist and Ivan D Chase. Data-based analysis of winnerloser models of hierarchy formation in animals. Bulletin of mathematical biology, 71(3):556-584, 2009.

[53] M. Massink and N. De Francesco. Modelling free flight with collision avoidance. In Proceedings Seventh IEEE International Conference on Engineering of Complex Computer Systems, pages 270-279, 2001.

[54] M. Mazzarello and E. Ottaviani. A traffic management system for realtime traffic optimisation in railways. Transportation Research Part B: Mathodological, 41:246-274, 2007.

[55] L. Meng and X. Zhou. Simultaneous train rerouting and rescheduling on an n-track network: A model reformulation with network-based cumulative flow variables. Transportation Research Part B: Methodological, 67:208-234, 2014.

[56] P. K. Menon, G. D. Sweriduk, and B. Sridhar. Optimal strategies for free flight air traffic conflict resolution. Journal of Guidance, Control, and Dynamics, 22:202-211, 1997.

[57] R.B. Myerson and M.A. Satterthwaite. Efficient mechanisms for bilateral trading. Journal of Economic Theory, 29(2):265-281, 1983.

[58] Wei Ni and Daizhan Cheng. Leader-following consensus of multiagent systems under fixed and switching topologies. Systems \& Control Letters, 59(3-4):209-217, 2010.

[59] Ernesto Nunes, Marie Manner, Hakim Mitiche, and Maria Gini. A taxonomy for task allocation problems with temporal and ordering constraints. Robotics and Autonomous Systems, 90:55-70, 2017.

[60] Mahito Okura. Coopetitive strategies of japanese insurance firms a game-theory approach. International Studies of Management \& Organization, 37(2):53-69, 2007.

[61] Mahito Okura, David Carfì, et al. Coopetition and game theory. Journal of Applied Economic Sciences, 9(3):29, 2014.

[62] Reza Olfati-Saber, J Alex Fax, and Richard M Murray. Consensus and cooperation in networked multi-agent systems. Proceedings of the IEEE, 95(1):215-233, 2007.

[63] ON-TIME Consortium. Tools for real-time perturbation management including human machine interface. Deliverable 4.2 of the ONTIME - Optimal Networks for Train Integration Management across Europe. Project funded by the European Union's Seventh Framework Programme, 2014.

[64] Lucia Pallottino, Vincenzo G Scordio, Antonio Bicchi, and Emilio Frazzoli. Decentralized cooperative policy for conflict resolution in multivehicle systems. IEEE Transactions on Robotics, 23(6):1170$1183,2007$.

[65] P. Pellegrini, L. Castelli, and R. Pesenti. Secondary trading of airport slots as a combinatorial exchange. Transportation Research Part E: Logistics and Transportation Review, 48(5):1009-1022, 2012.

[66] P. Pellegrini, G. Marlière, and J. Rodriguez. Optimal train routing and scheduling for managing traffic perturbations in complex junctions. Transportation Research Part B: Methodological, 59C:58-80, 2014.

[67] P. Pellegrini and J. Rodriguez. Single European Sky and Single European Railway Area: A system level analysis of air and rail transportation. Transportation Research Part A: Policy and Practice, 57:64-86, 2013.
[68] A. R. Pritchett and A. Genton. Negotiated decentralized aircraft conflict resolution. IEEE Transactions on Intelligent Transportation Systems, 19(1):81-91, 2018

[69] H. Provenca and E. Oliveira. An adaptive multi-agent system for railway traffic control. GESTS International Transactions on Computer Science and Engineering, 9(1), 2005. 12 pages.

[70] E. Quaglietta, P. Pellegrini, R.M.P. Goverde, T. Albrecht, B. Jaekel, G. Marlière, J. Rodriguez, T. Dollevoet, B. Ambrogio, D. Carcasole, M. Giaroli, and G. Nicholson. The ON-TIME real-time railway traffic management framework: A proof-of-concept using a scalable standardised data communication architecture. Transportation Research Part C: Emerging Technologies, 63:23-50, 2016.

[71] A. Reina, G. Valentini, C. Fernàndez-Oto, M. Dorigo, and V. Trianni. A design pattern for decentralised decision making. PLoS ONE, 10(10), 2015. e0140950.

[72] Wei Ren and Randal W Beard. Consensus seeking in multiagent systems under dynamically changing interaction topologies. IEEE Transactions on automatic control, 50(5):655-661, 2005.

[73] Simone C. Riedmiller and Martin A. Riedmiller. A neural reinforcement learning approach to learn local dispatching policies in production scheduling. In IJCAI, 1999.

[74] Marco Rinaldi, Willem Himpe, and Chris M.J. Tampère. A sensitivitybased approach for adaptive decomposition of anticipatory network traffic control. Transportation Research Part C: Emerging Technologies, 66:150 - 175, 2016.

[75] J. Rios-Torres and A. Malikopoulos. A survey on the coordination of connected and automated vehicles at intersections and merging at highway on-ramps. IEEE Transactions on Intelligent Transportation Systems, 18(5):1066-1077, 2017.

[76] Flávio Rodrigues, Victória Souza, and Joao Leitao. Strategic coopetition of global brands: a game theory approach to nike+ ipod sport kitco-branding. International Journal of Entrepreneurial Venturing, 3(4):435-455, 2011.

[77] G. Rodrigues de Campos, P. Falcone, R. Hult, H. Wymeersch, and J. Sjb̈erg. Traffic coordination at road intersections: Autonomous decision-making algorithms using model-based heuristics. IEEE Intelligent Transportation Systems Magazine, 9(1):8-21, 2017.

[78] Marcella Samà, Andrea D'Ariano, Francesco Corman, and Dario Pacciarelli. A variable neighbourhood search for fast train scheduling and routing during disturbed railway traffic situations. Computers \& Operations Research, 78:480 - 499, 2017.

[79] D. Sànchez, S. Martìnez, and J. Domingo-Ferrer. Co-utile ridesharing. In J. Domingo-Ferrer and D. Sànchez, editors, Co-utility. Studies in Systems, Decision and Control, number 110 in Studies in Systems, Decision and Control, pages 117-138. Springer Cham, 268 edition, 2018.

[80] Danny Schipper and Lasse Gerrits. Differences and similarities in european railway disruption management practices. Journal of rail transport planning \& management, 8(1):42-55, 2018.

[81] Thomas Schlechte. Railway Track Allocation: Models and Algorithms. $\mathrm{PhD}$ thesis, Technische Universitt Berlin, 2012.

[82] D. Šemrov, R. Marsetič, M. Žura, L. Todorovski, and A. Srdic. Reinforcement learning approach for train rescheduling on a singletrack railway. Transportation Research Part B: Methodological, 86:250 - 267, 2016.

[83] Fei Shang, Jingyuan Zhan, and Yangzhou Chen. Distributed model predictive control for train regulation in urban metro transportation. In 2018 21st International Conference on Intelligent Transportation Systems (ITSC), pages 1592-1597. IEEE, 2018.

[84] Arambam James Singh, Duc Thien Nguyen, Akshat Kumar, and Hoong Chuin Lau. Multiagent decision making for maritime traffic management. In Proceedings of the AAAI Conference on Artificial Intelligence, volume 33(01), pages 6171-6178, 2019.

[85] P. Su and B.B. Park. Auction-based highway reservation system an agent-based simulation study. Transportation Research Part C: Emerging Technologies, 60:211-226, 2015.

[86] Kian-Moh Terence Tan and John E Hiles. Modeling the human decision making process in maritime interdiction using conceptual blending theory. In Simulation Interoperability Standards Organization (SISO) papers and Behavior Representation in Modeling and Simulation (BRIMS), 2008.

[87] Teck-Hou Teng, Hoong Chuin Lau, and Akshat Kumar. Coordinating vessel traffic to improve safety and efficiency. In Proceedings of the 16th Conference on Autonomous Agents and MultiAgent Systems, pages 141-149. International Foundation for Autonomous Agents and Multiagent Systems, 2017. 
[88] Teck-Hou Teng, Hoong Chuin Lau, and Akshat Kumar. A multiagent system for coordinating vessel traffic. In Proceedings of the 16 th Conference on Autonomous Agents and MultiAgent Systems, pages 1814-1816. International Foundation for Autonomous Agents and Multiagent Systems, 2017.

[89] Sofie Van Thielen, Francesco Corman, and Pieter Vansteenwegen. Considering a dynamic impact zone for real-time railway traffic management. Transportation Research Part B: Methodological, 111:39 59, 2018.

[90] Yu-Ping Tian and Cheng-Lin Liu. Consensus of multi-agent systems with diverse input and communication delays. IEEE Transactions on Automatic Control, 53(9):2122-2128, 2008.

[91] C. Tomlin, G. J. Pappas, and S. Sastry. Conflict resolution for air traffic management: a study in multiagent hybrid systems. IEEE Transactions on Automatic Control, 43(4):509-521, 1998.

[92] Claire J. Tomlin, George J. Pappas, Jana Košecká, John Lygeros, and Shankar S. Sastry. Advanced air traffic automation: A case study in distributed decentralized control. In Bruno Siciliano and Kimon P. Valavanis, editors, Control Problems in Robotics and Automation, pages 261-295. Springer Berlin Heidelberg, Berlin, Heidelberg, 1998

[93] J. Törnquist Krasemann. Design of an effective algorithm for fast response to re-scheduling of railway traffic during disturbances. Transportation Research Part C: Emerging Technologies, 20:62-78, 2012.

[94] G. Valentini, E. Ferrante, and M. Dorigo. The best-of-n problem in robot swarms: Formalization, state of the art, and novel perspectives. Frontiers in Robotics and AI, 9, 2017. 9 pages.

[95] Fangliang Xiao, Han Ligteringen, Coen Van Gulijk, and Ben Ale. Artificial force fields for multi-agent simulations of maritime traffic: a case study of chinese waterway. Procedia Engineering, 45:807-814, 2012.

[96] Fangliang Xiao, Han Ligteringen, Coen Van Gulijk, and Ben Ale. Nautical traffic simulation with multi-agent system for safety. In 16th International IEEE Conference on Intelligent Transportation Systems (ITSC 2013), pages 1245-1252. IEEE, 2013.

[97] Zhe Xiao, Xiuju Fu, and Liye Zhang Loganathan Ponnambalamand Rick Siow Mong Goh. Data-driven multi-agent system for maritime traffic safety management. In 2017 IEEE 20th International Conference on Intelligent Transportation Systems, 2017. 6 pages.

[98] Cui Yong, Martin Ullrich, and Liang Jiajian. Decentralized, autonomous train dispatching using swarm intelligence in railway operations and control. In 7th International Conference on Railway Operations Modelling and Analysis - RailLille2017, pages 521-540. IAROR, 2017.

[99] J. J. Q. Yu, A. Y. S. Lam, and Z. Lu. Double auction-based pricing mechanism for autonomous vehicle public transportation system. IEEE Transactions on Intelligent Vehicles, 3(2):151-162, 2018.

[100] Zhicong Zhang, Li Zheng, and Michael X. Weng. Dynamic parallel machine scheduling with mean weighted tardiness objective by qlearning. The International Journal of Advanced Manufacturing Technology, 34(9):968-980, 2007. 